\title{
JunB potentiates function of BRCA1 activation domain 1 (AD1) through a coiled-coil-mediated interaction
}

\author{
Yan-Fen $\mathrm{Hu}$ and Rong $\mathrm{Li}^{1}$ \\ Department of Biochemistry and Molecular Genetics, School of Medicine, University of Virginia, Charlottesville, \\ Virginia 22908, USA
}

BRCA1 is involved in the regulation of multiple nuclear events including transcription. AD1, one of the two trans-activation domains in BRCA1, stimulates transcription in a cell context-dependent manner. Here, it is shown that BRCA1 interacts with Jun proteins via a coiled-coil motif in AD1 and the basic leucine zipper (bZIP) region of the Jun proteins. The Jun-interacting domain in BRCA1 is critical for AD1-mediated transcriptional activation. In particular, the strength of AD1 in transcriptional activation is limited by the JunB level and ectopic expression of JunB potentiates the transcriptional activity of AD1. Furthermore, JunB mRNA expression is down-regulated in many ovarian tumor tissues examined. Thus, the coiled-coil-mediated cooperation between BRCA1 and JunB may facilitate the function of these proteins in tissue-specific transcriptional regulation and tumor suppression.

[Key Words: BRCA1; AP1; Jun, transcription; bZIP; coiled-coil]

Received March 26, 2002; revised version accepted May 2, 2002.

Mutations in BRCA1 account for a significant proportion of hereditary breast and ovarian cancers (Welcsh and King 2001). Intense research in the past several years has implicated BRCA1 in regulation of multiple aspects of nuclear function including transcriptional activation, DNA repair, recombination, and checkpoint control (Zhang et al. 1998b; Monteiro 2000; Scully and Livingston 2000; Zheng et al. 2000; Parvin 2001). When tethered to a transcriptional promoter, the BRCA1 carboxyl terminus (BRCT) domain can stimulate transcription and remodel chromatin (Chapman and Verma 1996; Monteiro et al. 1996; $\mathrm{Hu}$ et al. 1999). The functional relevance of these studies is underscored by the observation that cancer-predisposing mutations in the same region abolish the activity of the BRCT domain in transcription and chromatin remodeling. Consistent with its potential role in transcriptional regulation, BRCA1 is associated with the RNA polymerase II holoenzyme and chromatin-modifying proteins (Scully et al. 1997; Neish et al. 1998; Yarden and Brody 1999; Bochar et al. 2000; Pao et al. 2000). In addition, the full-length BRCA1 protein can potentiate transcription from several natural promoters (Somasundaram et al. 1997; Ouchi et al. 1998; Zhang et al. 1998a; Harkin et al. 1999; MacLachlan et al. 2000).

In addition to the BRCT domain (AD2), a second transactivation domain of BRCA1 (AD1) was recently discov-

${ }^{1}$ Corresponding author.

E-MAIL r12t@virginia.edu; FAX (434) 924-5069.

Article and publication are at http://www.genesdev.org/cgi/doi/10.1101/ gad.995502. ered (Hu et al. 2000; see Fig. 1A). Moreover, a highly conserved coiled-coil motif in AD1 is critical for its function in transcriptional activation. Interestingly, this coiled-coil region is located immediately upstream of a demarcation point for cancer-predisposing mutations of $B R C A 1$ in which, according to a phenotype-genotype correlation study, change in ovarian cancer risks occurs (Gayther et al. 1995). Therefore, it has been suggested that this region of the protein may contain a functional domain that specifically protects ovarian epithelial cells from developing tumors (Rahman and Stratton 1998).

In comparison with $\mathrm{AD} 2$, transcriptional activation by $\mathrm{AD} 1$ is cell-type dependent and less robust $(\mathrm{Hu}$ et al. 2000). In some cell lines (e.g., ES2, an ovarian cancer cell line), $\mathrm{AD} 1$ by itself exhibits very modest transcriptional activity, but it can synergistically stimulate transcription with $\mathrm{AD} 2$, whereas in other cell lines (e.g., HEK293T, an embryonic kidney cell line), AD1 does not confer transcriptional stimulation, either alone or with AD2. The molecular basis for the cell-context dependence of AD1 activity remains to be elucidated, but one likely possibility is that a putative partner(s) that mediates $\mathrm{AD} 1$ function may be limiting in these cells.

Herein, we find that AD1 interacts with the Jun proteins of the AP1 family. We show that the BRCA1-Jun interaction is mediated by the coiled-coil region of BRCA1 and the bZIP region of the Jun proteins. Furthermore, the cellular level of JunB is an important determinant for the potency of AD1 in transcriptional activation. We show that the mRNA level of JunB is downregulated in the majority of ovarian tumor tissues 
$\mathbf{A}$

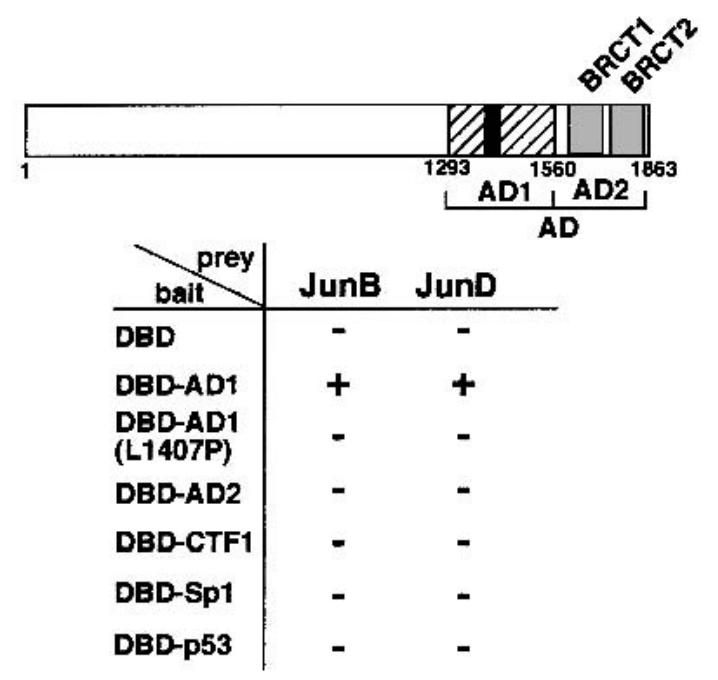

B

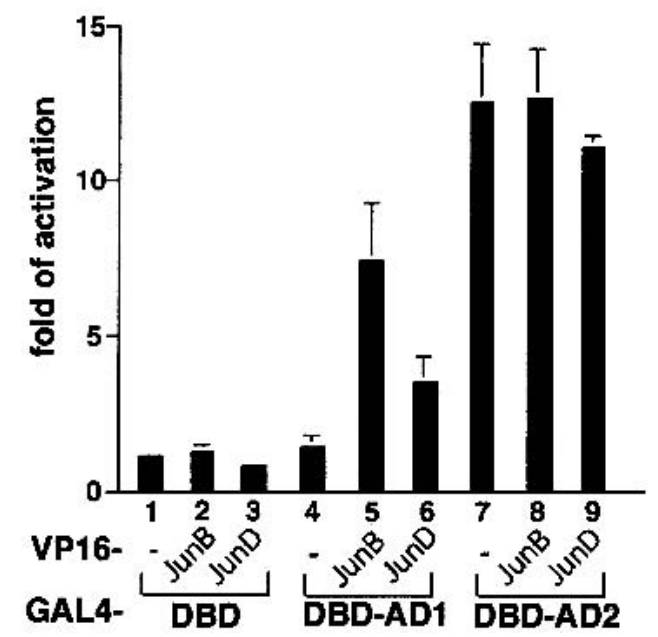

Figure 1. BRCA1 interacts with JunB and JunD in yeast and mammalian two-hybrid assays. (A) Summary of the results from the yeast two-hybrid screen. The plus sign $(+)$ indicates rapid growth of the yeast cells in the selective medium as a result of elevated expression of the HIS3 gene. Also presented is a schematic diagram of the BRCA1 protein, illustrating the location of the trans-activation domains. The solid bar within AD1 indicates the coiled-coil motif. (B) Mammalian two-hybrid assay showing the interactions between BRCA1 and the Jun proteins. HEK293T cells were transfected with the mammalian bait and prey constructs. Shown in the y axis is the fold of increase in the luciferase activity over the negative control (column 1; GAL4DBD and VP16).

examined. Thus, the physical and functional link between BRCA1 and JunB may be important for their functions in tissue-specific transcriptional regulation and suppression of tumor development.

\section{Results}

JunB and JunD interact with AD1 of BRCA1 in the yeast and mammalian two-hybrid systems

To identify the potential partner(s) of BRCA1 that mediate $\mathrm{AD} 1$ function in transcriptional activation, we con- ducted a yeast two-hybrid screen using AD1 as the bait and a prey library of human ovary cDNA. To reduce the high background due to the intrinsic transcriptional activity of AD1, $100 \mathrm{mM}$ 3-aminotriazole (3-AT) was included in the medium throughout the screen. A largescale screen uncovered multiple independent cDNA clones that encode human JunB and JunD, two members of the activation protein 1 (AP1) family (Fig. 1A). The AP-1 family of transcription factors consists of homodimers and heterodimers of the Jun and Fos subfamilies that play important roles in diverse aspects of cell proliferation and differentiation (Mechta-Grigoriou et al. 2001; Shaulian and Karin 2001). Mammalian Jun proteins include c-Jun, JunB, and JunD; Fos proteins include c-Fos, FosB, Fra1, and Fra2. A common characteristic of the AP1 family members is the presence of the basic leucine zipper (bZIP) motif that serves as the DNA-binding and dimerization domains (Chinenov and Kerppola 2001).

The interactions between $\mathrm{AD} 1$ and the Jun proteins detected in the yeast system were specific, as JunB and JunD failed to bind to other known trans-activation domains, including AD2 of BRCA1, and the activation domains of several other mammalian transcription factors such as p53, Sp1, and CTF1 (Fig. 1A). Previous work has shown that a mutation at one of the key leucine residues in the coiled-coil motif in AD1 (L1407P) abolishes its function in transcriptional activation (Hu et al. 2000). The same mutation also abrogated the ability of AD1 to bind to the Jun proteins (Fig. 1A). Interestingly, all partial cDNA clones of JunB and JunD isolated from the screen encode the bZIP domain, suggesting that the AD1-Jun interaction may be mediated by the coiled-coil region of BRCA1 and the bZIP region of the Jun proteins.

To confirm the yeast two-hybrid results, we also used a mammalian two-hybrid assay in which GAL4-AD1 was coexpressed with JunB or JunD that was fused to a potent transcriptional activation domain (VP16). Consistent with previous findings (Hu et al. 2000), GAL4-AD1 alone did not significantly activate transcription in HEK293T cells (Fig. 1B, cf. column 1 with 4), whereas GAL4-AD2 functioned as a potent activator in the same cellular context (Fig. 1B, column 7). Coexpression of VP16-JunB resulted in a significant elevation of transcriptional activation by GAL4-AD1 (Fig. 1B, cf. column 4 with 5). VP16-JunD also had a similar, albeit less pronounced, effect on GAL4-AD1 (Fig. 1B, column 6). In contrast, the same prey constructs did not enhance the activity of GAL4-DBD (Fig. 1B, columns 1-3) or GAL4AD2 (Fig. 1B, columns 7-9). Expression of the GAL4 derivatives was unaffected by the VP16-Jun proteins (data not shown). Thus, the results from both yeast and mammalian two-hybrid systems indicate that JunB and JunD interact with $\mathrm{AD} 1$ in vivo.

BRCA1 specifically interacts with the Jun proteins of the AP1 family

To verify the two-hybrid findings, we examined the ability of various AP1 family members to interact with the 
native full-length BRCA1 in human cells. FLAG-tagged c-Jun, JunB, JunD, and c-Fos were expressed in HEK293T or ES2 cells (Fig. 2A, top). Following immunoprecipitation with an anti-FLAG antibody, the presence of the endogenous BRCA1 in the immunoprecipitates was detected by immunoblotting with an anti-BRCA1 antibody (Fig. 2A). Consistent with the two-hybrid results, the FLAG-tagged JunB and JunD were associated with native BRCA1 (Fig. 2A, lanes 2 and 4). In addition, BRCA1 was also coprecipitated with the FLAG-tagged c-Jun (Fig. 2A, lane 3). The in vivo association of BRCA1 and the Jun proteins was unlikely to be mediated by nucleic acids, as it was not affected by the treatment of nuclease or ethidium bromide (data not shown).

In contrast to the Jun proteins, the FLAG-tagged c-Fos did not bind to native BRCA1 in either HEK293T (Fig. 2A, lane 5) or ES2 cells (Fig. 2A, lane 9). For reasons that will become obvious later, the co-IP experiment in HEK293T cells was also repeated in the presence of ectopically expressed HA-JunB. Once again, no endogenous BRCA1 was detected in the FLAG-cFos immunoprecipitate (Fig. 2A, lane 6). It is known that c-Fos forms heterodimers with the Jun proteins, but not homodimers with itself in vivo (Karin et al. 1997). Endogenous Jun proteins were coimmunoprecipitated with the FLAGcFos (data not shown). Thus, our finding suggests that BRCA1 does not bind to either c-Fos monomers or JunFos heterodimers.

Next, we sought to ascertain the interaction between BRCA1 and the Jun proteins in a more direct manner. The bZIP region of various AP1 proteins was fused with glutathione S-transferase (GST). The purified GST proteins were immobilized on glutathione beads and incubated with the in vitro translated, ${ }^{35}$ S-labeled AD fragment of BRCA1. As shown in Figure 2B, AD was pulled down by all three GST-Jun fusion proteins (Fig. 2B, lanes 2-4), but not by GST alone (Fig. 2B, lane 1) or GST-cFos (Fig. 2B, lane 5). This result suggests a direct interaction between BRCA1 and the Jun proteins. Furthermore, the in vitro finding confirms that the bZIP region of the Jun proteins is sufficient for binding to BRCA1.

To ascertain the BRCA1-Jun interaction in a more physiological context, the endogenous AP1 proteins from ES2 cells was immunoprecipitated with various commercially available antibodies. Subsequent immunoblotting with an anti-BRCA1 antibody showed that the endogenous BRCA1 was coprecipitated with both c-Jun and JunB, but not c-Fos (Fig. 2C, cf. lanes 2, 4, and 6-8). In addition, the BRCA1 signals were diminished when two antibody-specific competing peptides were included in the immunoprecipitation reactions (Fig. 2C, cf. lane 2 with 3 and 4 with 5). A reciprocal co-IP experiment using two different anti-BRCA1 antibodies also shows the physical association between BRCA1 and c-Jun. Interestingly, the BRCA1-Jun interaction is refractory to a fairly high-salt and detergent concentration (500 $\mathrm{mM} \mathrm{NaCl}$ and $1 \% \mathrm{NP}-40$ ). Taken together, the results strongly indicate an in vivo association of BRCA1 with specific members of the AP1 family.
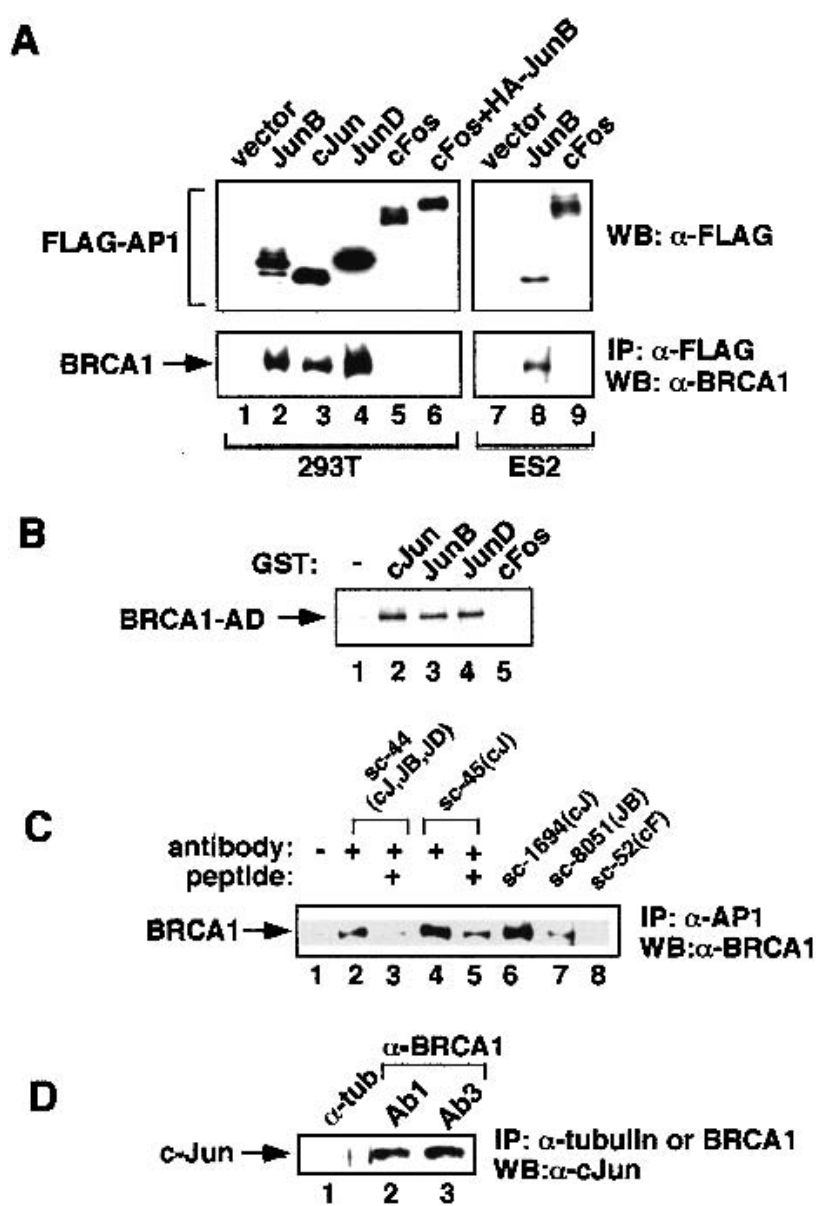

Figure 2. Interaction of the Jun proteins with BRCA1. (A) Human HEK293T (lanes 1-6) or ES2 (lanes 7-9) cells were transfected with the various expression vectors for the FLAG-tagged AP-1 proteins. Following immunoprecipitation (IP) with an anti-FLAG antibody, the proteins in the immunoprecipitates were resolved by SDS-PAGE, and the presence of the endogenous BRCA1 proteins was detected by immunoblotting (WB) with an anti-BRCA1 antibody (bottom). Equal amounts of the cell lysates were analyzed by immunoblotting for the expression of the FLAG-tagged AP-1 proteins (top). Lane 6 shows that c-Fos does not interact with BRCAl, even in the presence of exogenous HA-tagged JunB. (B) ${ }^{35}$ S-labeled BRCA1-AD was made with an in vitro translation kit (Promega), and incubated with the GST alone (lane 1) or GST fused with the bZIP region of various AP1 proteins (lanes 2-5) that were immobilized on glutathione beads. After extensive washing, the coprecipitated proteins were analyzed by SDS-PAGE and fluorography. $(C)$ Coimmunoprecipitation of native BRCA1 and native Jun proteins in ES2 cells. Lysates of ES2 cells were immunoprecipitated with different anti-AP1 antibodies (sc-44 for pan-Jun, sc-45 and sc1694 for c-Jun, sc-8051 for JunB, and sc-52 for c-Fos). The presence of BRCA1 in the immunoprecipitates was detected by Western blotting using an anti-BRCA1 antibody (Ab-1 from Oncogene). In lanes 3 and 5, an excess of the corresponding competing peptides was included in the immunoprecipitation reactions. (D) A reciprocal co-IP was performed in ES2 cell lysates using either anti- $\alpha$-tubulin (as a negative control) or antiBRCA1 antibodies (Ab1 and Ab3; Oncogene) in the immunoprecipitation. The blot was probed with an anti-cJun antibody (sc-1694). 
AD1 is necessary and sufficient for BRCA1 binding to the Jun proteins

To further characterize the Jun-binding domain in BRCA1, we constructed a series of deletional mutants and analyzed their ability to interact with the FLAGJunB using the coimmunoprecipitation assay. The majority of cancer-predisposing mutations in BRCA1 result in truncation of the protein. As summarized in Figure 3, a disease-associated BRCA1 truncation mutant that retained the coiled-coil region in $\mathrm{AD} 1$ still interacted with BRCA1 (Fig. 3, construct 2), whereas those that lacked the AD1 region failed to bind to JunB (Fig. 3, constructs 3 and 4). In a different set of mutational analyses, multiple fragments that spanned the entire BRCA1 protein were tested for JunB binding (Fig. 3, constructs 5-12). The only fragments that displayed significant affinity for JunB were those that contained AD1 (Fig. 3, constructs 10 and 12). Further deletional studies within AD1 (Fig. 3, constructs 13-16) revealed a minimal JunB-binding domain (Fig. 3, construct 15; amino acids 1343-1440), which consists of the coiled-coil motif and an $\sim 60$ amino acid upstream sequence. This finding indicates that the $\mathrm{AD} 1$ region is both necessary and sufficient for BRCA1 binding to JunB.

The coiled-coil motif in BRCA1 is critical for binding to Jun and for AD1-mediated transcriptional activation

To establish a stronger link between the BRCA1-Jun interaction and $\mathrm{AD} 1$-dependent transcriptional activation,

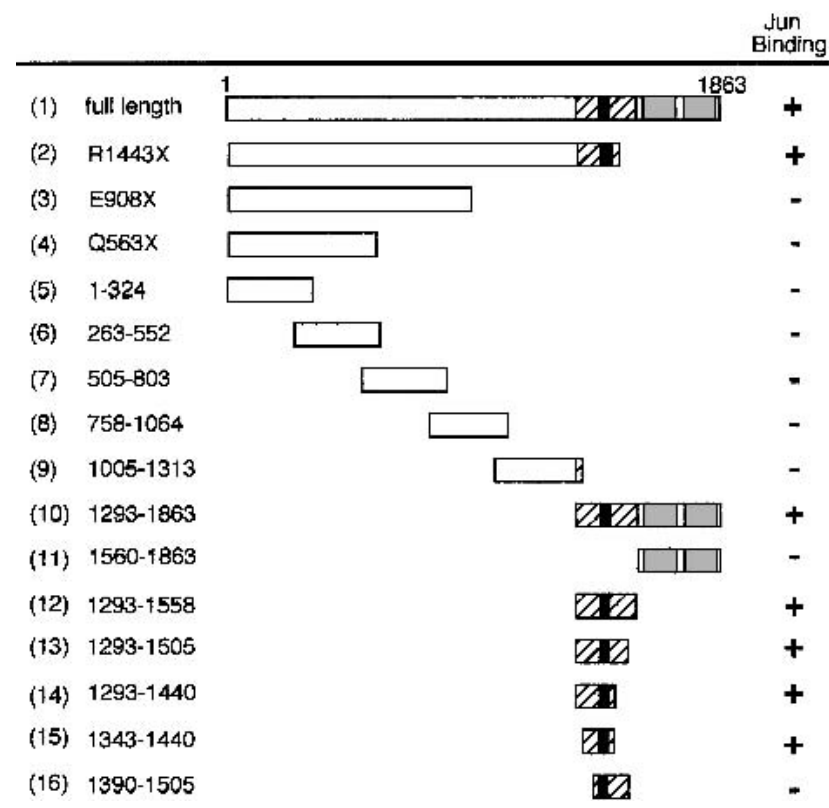

Figure 3. Characterization of the Jun-binding domain in BRCA1. Various HA-tagged BRCA1 fragments were ectopically coexpressed with FLAG-JunB in HEK293T cells. Immunoprecipitation was carried out using an anti-FLAG antibody. The plus sign indicates a significant signal of the BRCA1 fragments in the anti-FLAG immunoprecipitates. The hatched box indicates AD1, whereas the shaded boxes designate the two BRCT repeats in $\mathrm{AD} 2$. The solid bar within $\mathrm{AD} 1$ represents the coiled-coil region.

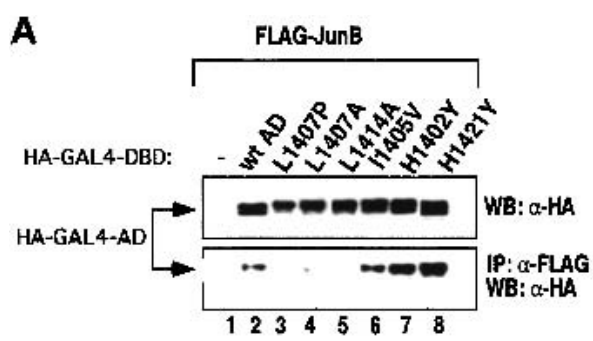

B
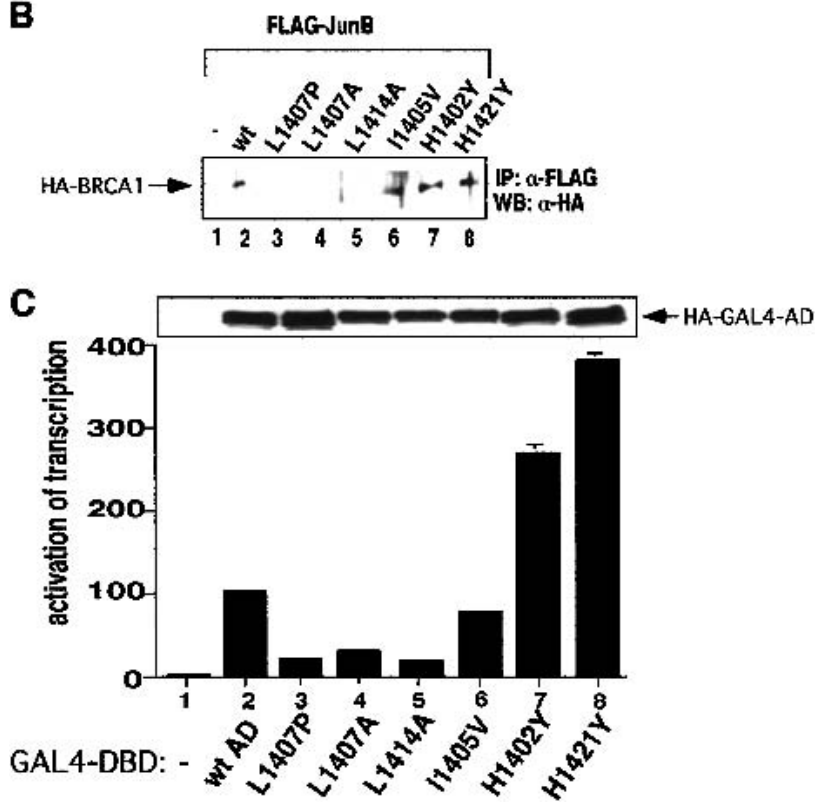

Figure 4. BRCA1-Jun interaction correlates with AD1-mediated transcriptional activation. (A) HEK293T cells were cotransfected with the expression vectors for the FLAG-tagged JunB and HA-tagged GAL4-AD domain. Lysates from the transfected cells were immunoprecipitated (IP) with an anti-FLAG antibody, and the immunoprecipitates were probed by immunoblotting (WB) with an anti-HA antibody (bottom). As a control, expression of the wild-type and mutant GAL4-AD fusion proteins was detected by immunoblotting of the crude lysates using the anti-HA antibody $(t o p)$. (B) HA-tagged full-length BRCA1 proteins were coexpressed with FLAG-JunB in HEK293T cells. Anti-FLAG immunoprecipitation was followed by immunoblotting using the anti-HA antibody. $(C)$ ES-2 cells were cotransfected with the GAL4-AD expression vectors and a GAL4-responsive luciferase reporter plasmid. Also shown at top of the graph is a Western blot for the wild-type and mutant fusion proteins. The relative transcriptional activity of the wildtype GAL4-AD construct is set at 100 .

we introduced various point mutations into the coiledcoil region of AD1 (Fig. 4). All mutants were expressed at similar levels as the wild-type proteins (Fig. 4A,C). The mutational effect on the BRCA1-JunB interaction was tested in the contexts of both AD (Fig. 4A) and the fulllength BRCA1 (Fig. 4B). Extensive work on other coiledcoil proteins has shown that the leucine residues at position $\mathrm{d}$ of the heptad repeat are the critical determinants for the coiled-coil structure (Lupas et al. 1991; Lupas 1996). Substitution of two such leucines in the coiled- 
coil region of BRCA1 (L1407 and L1414) abrogated the BRCA1-JunB interaction (Fig. 4A,B, lanes 3-5). Importantly, the same mutations also impaired the transcriptional activity of GAL4-AD in the luciferase reporter assay (Fig. 4C, lanes 3-5). In contrast, mutations that presumably did not affect the coiled-coil structure (I1405V, H1402Y, and H1421Y) still retained the affinity of BRCA1 for JunB (Fig. 4A,B, lanes 6-8). Likewise, the GAL4 fusion proteins carrying these mutations were still capable of stimulating transcription (Fig. 4C, lanes 6-8). All of the mutations shown in Figure 4 had the same effect on c-Jun and JunD binding as they did on JunB (data not shown). Thus, the affinity of BRCA1 for the Jun proteins strongly correlates with the strength of the trans-activation domain in transcriptional stimulation.

\section{JunB potentiates $A D 1$ function in transcriptional activation}

Previous characterization of AD1 indicates that this trans-activation domain functions in a cell context-dependent manner and that it displays a less robust transcription activity than AD2. For example, HEK293T cells were deficient in AD1-mediated transcriptional activation, despite their ability to support high-transfection efficiency and robust expression of the GAL4 derivatives (Hu et al. 2000). Given the specific interactions between $\mathrm{AD} 1$ and the Jun proteins, we speculated that the lack of AD1 function in HEK293T cells might be due to limited expression of one or more Jun proteins. Immunoblotting of crude lysates from HEK293T cells indicated that the protein level of JunB was extremely low (Fig. 5A, lane 2), as has been observed by others (Bakiri et al. 2000). In contrast, the levels for c-Jun, JunD, and c-Fos in HEK293T cells were comparable with those in the other cell lines examined (Fig. 5A).

To determine whether the low level of JunB protein in HEK293T cells was causally related to the lack of AD1 activity, we asked whether the deficiency in supporting AD1 function could be rescued by ectopic expression of JunB. As shown in Figure 5B, coexpression of FLAGJunB and GAL4-AD1 significantly enhanced the ability of AD1 to activate transcription (Fig. 5B, cf. lanes 6 and 7). However, JunB did not superactivate GAL4-AD2 (Fig. $5 \mathrm{~B}$, cf. lanes 11 and 12 ), nor did it rescue the transcriptional defect of a coiled-coil mutant of AD1 that failed to bind JunB (L1407P; Fig. 5B, cf. lanes 16 and 17). These results strongly indicate that JunB potentiates BRCA1 function through its interaction with the coiled-coil region of $\mathrm{AD} 1$. In contrast to JunB, ectopic expression of c-Jun, JunD, or c-Fos failed to complement the deficiency of HEK293T cells (Fig. 5B, lanes 8-10), despite their expression levels equivalent to that of junB (Fig. 2A). This differential effect of the AP1 proteins was observed at multiple concentrations of the AP1 expression vectors (data not shown). Thus, although all three Jun proteins are capable of binding to BRCA1, JunB exhibits a distinct function in facilitating AD1-mediated transcriptional activation.

Although JunB protein was expressed above the detect- able level in most of the cell lines examined (Fig. 5A), it could still be limiting for supporting AD1-mediated transcriptional activation. For example, despite the higher JunB expression in ES2 cells than that in HEK293T cells, $\mathrm{AD} 1$ alone only moderately activates transcription in ES2 cells ( $\mathrm{Hu}$ et al. 2000) (Fig. 5C, cf. lanes 1 and 6). Ectopic expression of JunB, and JunD to a lesser extent, enhanced $\mathrm{AD} 1-$ mediated transcriptional activation in ES2 cells (Fig. 5C, columns 7 and 9). As observed in HEK293T cells, c-Jun and c-Fos failed to confer such superactivation of AD1 (Fig. 5C, columns 8 and 10). Therefore, our data indicate that the strength of $\mathrm{AD} 1$ in transcriptional activation is limited by the cellular level of JunB. An interesting difference between HEK293T and ES2 cells with regard to AD1 function is that ES2, but not HEK293T cells, can support a synergistic action of $\mathrm{AD} 1$ and $\mathrm{AD} 2$ (Hu et al. 2000). It is therefore plausible that the level of JunB in ES2 cells, although insufficient for supporting maximal activation by $\mathrm{AD} 1$ alone, may be replete for the cooperative activation by $\mathrm{AD} 1$ and $\mathrm{AD} 2$.

Given the proximity of the Jun-binding domain to the previously defined transition point of BRCA1 mutations that are associated with higher ovarian cancer risk (Gayther et al. 1995), we speculated that this domain likely provides special protective functions against ovarian cancer. In such an event, a deficit of JunB in ovarian epithelium might contribute to development of ovarian cancer in particular. To test this possibility, we compared the JunB mRNA level in tumor and normal tissues from the same individuals. The normalized real-time PCR results are shown in Figure 5D. In seven of the nine matched cDNA pairs from ovary tissue, JunB expression is significantly lower in tumor than in the normal issues (Fig. 5D, pairs $1-5,7$, and 8). The remaining two ovarian pairs (Fig. 5D, pairs 6 and 9) had very low JunB mRNA levels even in the normal tissues, suggesting that there might be some intrinsic abnormality in these two normal cases. Interestingly, the differential expression of JunB in most ovarian pairs was not obvious in a panel of matched cDNA pairs from breast tissue (Fig. 5D, pairs 10-16). This finding is consistent with the notion that the BRCA1-JunB interaction may play a role in specific suppression of ovarian cancer development.

\section{Discussion}

A wealth of evidence strongly suggests that BRCA1 plays an important role in the maintenance of genome stability via its function in transcriptional regulation and DNA repair. However, it remains puzzling that disease-associated mutations in BRCA1, which compromise such universal nuclear functions as transcription and DNA repair, specifically lead to elevation of the risk in developing breast and ovarian cancers. It has been suggested that the rapid proliferating status of the breast epithelium during puberty could render it particularly susceptible to BRCA1 mutation-dependent tumorigenesis (Scully and Livingston 2000). In addition, the fact that breast and ovary are both estrogen-responsive tissues and that BRCA1 can modulate transcriptional activation by estrogen receptor could also 

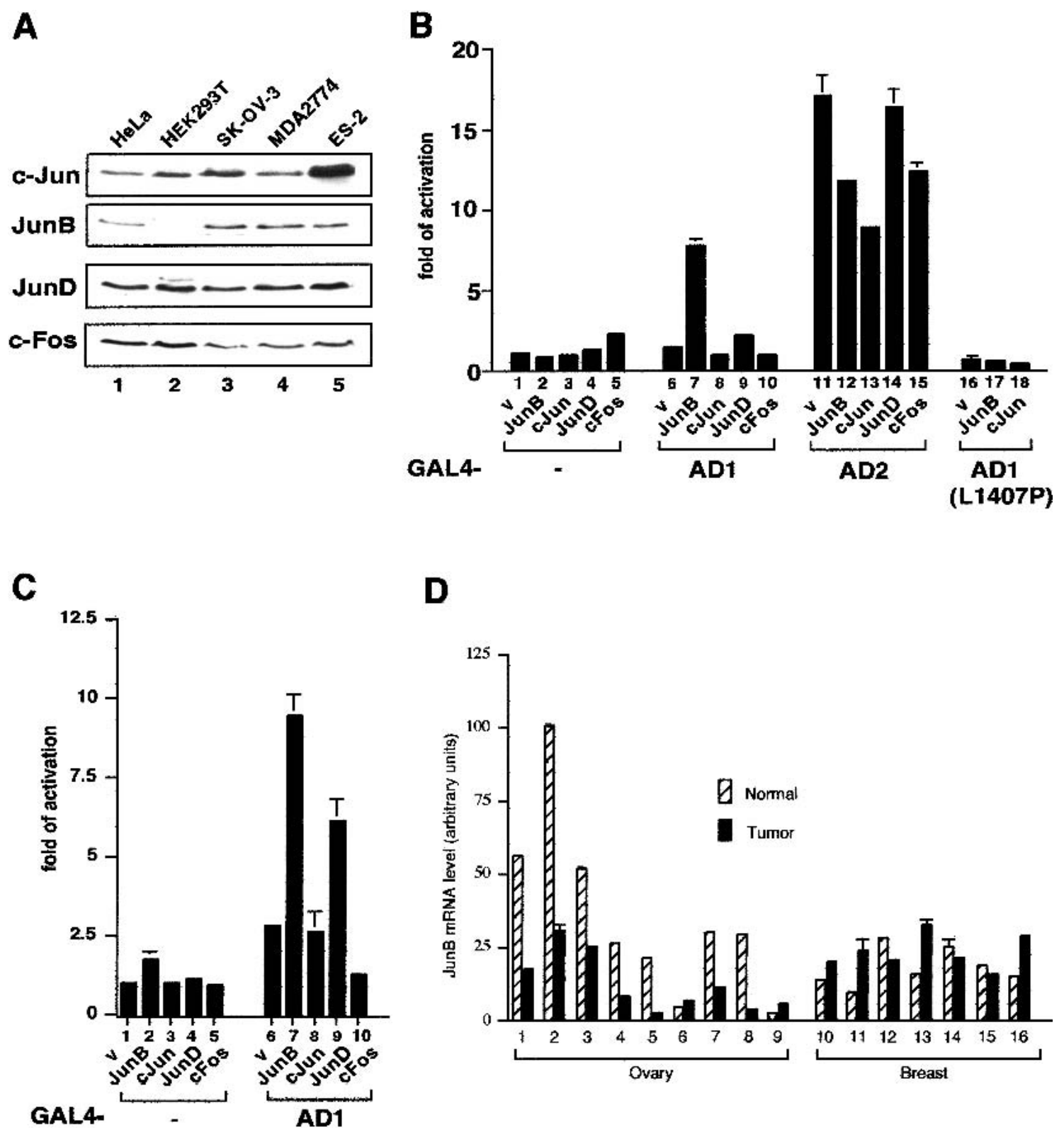

D

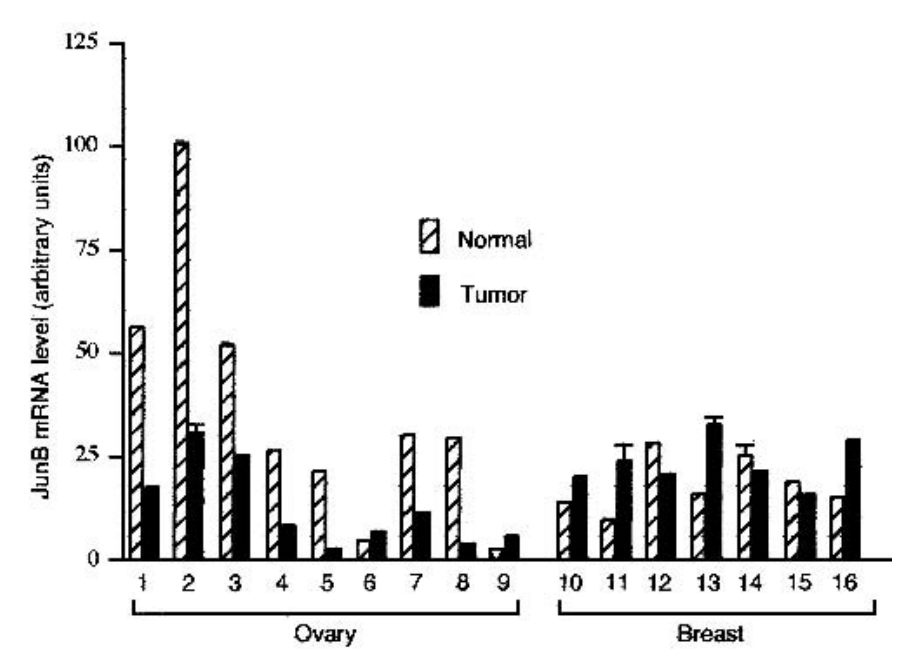

Figure 5. JunB can superactivate AD1-dependent transcriptional activation. $(A)$ An equal amount of cell lysates from various cell lines was probed by immunoblotting with specific antibodies against c-Jun, JunB, JunD, and c-Fos. (B) HEK293T cells were cotransfected with the luciferase reporter plasmid, the GAL4 derivatives, and various AP1 expression vectors. The relative luciferase activity in the presence of GAL4-DBD alone (column 1) is set at one. $(C)$ Luciferase reporter assay was performed in ES2 cells in the same manner as described in $B$. (D) Normalized matched cDNA pairs of normal and tumor ovarian (1-9) or breast (10-16) tissues were analyzed by the real-time PCR reactions for JunB mRNA expression.

explain the organ-specific nature of the BRCA1-dependent cancer risk (Fan et al. 1999, 2001; Zheng et al. 2001). It is also possible that the tissue-specific action of BRCA1 may be determined by more than one BRCA1-associated protein complex. In such an event, changes in the level and/or biochemical properties of a number of the BRCA1-associated proteins could contribute to the development of neoplasm in these tissues.

The findings in the current study strongly suggest that JunB plays an important role in mediating the function of one of the trans-activation domains of BRCA1 (AD1). First, both in vitro and in vivo experiments indicate that the two proteins interact through the coiled-coil domain of BRCA1 and the bZIP domain in JunB. The affinity of BRCA1 for JunB is strongly correlated with the strength of the AD1 domain in transcriptional activation. Furthermore, the data suggest that the cellular level of the JunB protein is an important determinant for AD1 function in transcriptional activation. Limited AD1 transcriptional activity due to a deficit of JunB can be rescued 
by ectopic expression of JunB. This finding provides a molecular explanation for the previously observed celltype dependent behavior of AD1 (Hu et al. 2000). Lastly, JunB mRNA levels are down-regulated in many ovarian tumor tissues compared with the normal tissues from the same individuals. Given that the AD1 domain of BRCA1 encompasses the demarcation point for BRCA1 mutations that are associated with increased risk of ovarian cancer, our results raise an intriguing possibility that the BRCA1-JunB interaction may be particularly important for suppression of ovarian cancer. However, due to the lack of detailed information concerning the clinical characteristics of the patients and the exact cell types from which the cDNA pairs were prepared, further study is needed to explore the exact biological significance of the differential JunB expression observed in the current work.

The exact geometry and stoichiometry of the BRCA1Jun complexes remain to be determined. It is also possible that the BRCA1-Jun interaction is aided by additional factors. The finding that BRCA1 is not associated with the cFos-containing complexes strongly suggests that Jun-Fos dimers, which is the predominant heterodimeric form of the AP1 family in vivo, are not capable of binding to BRCA1. This raises the possibility that BRCA1 may specifically target Jun monomers and/or Jun-Jun dimers. Conceivably, this Jun-specific interaction may lead to changes in the relative abundance, subnuclear localization, and biochemical characteristics of various forms of the AP1 proteins. The bZIP sequences among the Jun proteins are highly conserved, whereas those of Fos and Jun are relatively divergent. This could explain the disparity in their binding affinity for BRCA1. It is tempting to speculate that the leucine zipper motif may only provide the architectural basis for binding to BRCA1. Additional amino acid residues in the bZIP region that are unique to the Jun proteins may serve as the actual contact points for the coiled-coil region of BRCA1.

Whereas all three Jun proteins are capable of interacting with BRCA1, only JunB exhibits a strong enhancing effect on the AD1 transcriptional activity. Such a functional difference among the Jun proteins could be attributed to the more divergent sequences outside of the bZIP domain. The observation that BRCA1 can selectively target specific members of the AP1 family for physical and functional interaction may have profound biological ramifications. AP-1 family members form a large number of homodimers and heterodimers in vivo, each of which may exhibit distinct regulatory properties (Karin et al. 1997). As a consequence, different AP1 family members can play diverse and even opposing roles in cell proliferation and differentiation. For example, it has been well documented that c-Jun is positively involved in cell proliferation and Ras-mediated oncogenesis (Mechta-Grigoriou et al. 2001; Shaulian and Karin 2001). On the other hand, JunB and JunD can suppress Ras- and Src-induced cellular transformation (Johnson et al. 1996; Mechta et al. 1997). Moreover, JunB can antagonize the stimulatory function of c-Jun in cyclin D1 transcription
(Bakiri et al. 2000), and at the same time activate the transcription of $\mathrm{p} 16^{I N K 4 a}$, an inhibitor of the cyclin D-CDK4-D-CDK6 complexes (Passegue and Wagner 2000). Recent work with conditional JunB knockout mice also supports a role of JunB in tumor suppression (Passegue et al. 2001). Thus, although our study does not exclude a potential functional link between BRCA1 and the other two Jun proteins, the cooperation between BRCA1 and JunB in transcriptional regulation may be related to their known functions in suppression of tissue-specific tumor development.

\section{Materials and methods}

\section{Yeast two-hybrid screen}

The standard yeast two-hybrid screen was performed in the following manner. First, bait plasmid was generated by inserting a PCR-amplified cDNA fragment encoding AD1 (amino acids 1293-1559) of BRCA1 into pGBT8 (Clontech), resulting in an in-frame fusion with the GAL4 DNA-binding domain (DBD). Second, the resultant plasmid, pGBT8-AD1, and a human ovary cDNA library (Clontech) were cotransformed into the Saccharomyces cerevisiae reporter strain Hf7C according to the manufacturer's instructions (Clontech). Transformants were plated on synthetic medium lacking tryptophan, leucine, and histidine, but containing $100 \mathrm{mM}$ 3-aminotriazole (3-AT), which was used to suppress the relatively high background due to the intrinsic transcripional activity of the bait construct. Approximately 26 million transformants were screened, of which 32 were judged to be strongly HIS-positive. Additional screens using a number of negative controls were carried out (see Fig. 1A), and only those candidates exhibiting AD1-specific interaction were characterized further. Twenty clones contained partial cDNA sequences for JunD, and six contained the JunB cDNA sequences.

\section{Mammalian cell transfection and luciferase assay}

HEK293T cells were maintained in DMEM medium with 10\% fetal calf serum and were transfected using LipofectAmine 2000 (GIBCO BRL). ES-2 cells were grown in McCoy's 5A medium with $10 \%$ fetal calf serum and were transfected using LipofectAmine Plus (GIBCO BRL). In a typical GAL4-based transcription reporter assay performed in HEK293T cells, $0.5 \mu \mathrm{g}$ reporter and $1.0 \mu \mathrm{g}$ protein expression vectors were used. Half of these amounts were used for the luciferase assays in ES2 cells. The luciferase assays were performed as described previously (Hu et al. 2000). The expression vectors for GAL4 derivatives used in the mammalian two-hybrid assay were described previously (Hu et al. 2000). The vectors for the VP16 fusion proteins used in the mammalian two-hybrid assay were constructed by cloning the cDNA sequences for JunB and JunD into the prey plasmid as described previously (Yu et al. 1998).

\section{Coimmunoprecipitation}

The FLAG-tagged AP1 proteins were expressed from the pcDNA3 vector (Clontech). Plasmids pCG-HA-GAL4(1-94)-AD (Hu et al. 2000) and pcDNA3 3 -HA-BRCA1 (full length) (Scully et al. 1997) were described previously. Twenty-four hours after transfection, cells were washed twice with PBS and lysed in 0.5 $\mathrm{mL}$ Lysis Buffer (50 mM HEPES at $\mathrm{pH} 8.0,250 \mathrm{mM} \mathrm{NaCl}$, $0.1 \%$ NP-40, and protease inhibitor tablets from Roche- 
Bohringer). After brief sonication, the lysate was centrifuged at $14,000 \mathrm{rpm}$ for $12 \mathrm{~min}$ at $4^{\circ} \mathrm{C}$. The supernatant was used for subsequent coimmunoprecipitation. Fifteen microliters of $50 \%$ slurry of the anti-FLAG agarose beads (Sigma) were used in each immunoprecipitation, and the tubes were rotated overnight at $4^{\circ} \mathrm{C}$. Following incubation, the beads were centrifuged at 3300 rpm for $2 \mathrm{~min}$, and washed once with the lysis buffer and twice with a washing buffer (50 mM HEPES at $\mathrm{pH} 8.0,500 \mathrm{mM} \mathrm{NaCl}$, $1 \%$ NP-40), with each wash lasting at least $1 \mathrm{~h}$. The precipitates were then eluted in $10 \mu \mathrm{L} 2 \times$ protein sample buffer and loaded on SDS-polyacrylamide, followed by Western blotting according to the standard procedures. Five microliters of the input crude extract were used for detecting protein expression levels. The presence of the endogenous BRCA1 was detected using a commercially available anti-BRCA1 antibody (Ab- 1 from Oncogene). The HA-tagged proteins were detected using an anti-HA monoclonal antibody (12CA5).

The coimmunoprecipitation experiment shown in Figure 2C was conducted using lysates from ES2 cells, anti-Jun antibodies for immunoprecipitation, and an anti-BRCA1 antibody (Ab-1 from Oncogene) for immunoblotting. A total of $1.5 \mu \mathrm{g}$ of the following commercially available anti-AP1 antibodies (Santa Cruz Biotech.) were used in immunoprecipitation: sc-44 (anticJun, JunB, and JunD); sc-45 (anti-cJun); sc-1694 (anti-cJun); sc8051 (anti-JunB); sc-52 (anti-cFos). Two competing peptides for c-Jun in immunoprecipitation (sc-44p and sc-52p; Santa Cruz Biotech.) were used. The reciprocal co-IP shown in Figure 2D was done using $2.5 \mu \mathrm{g}$ of anti-BRCA1 (Ab1 and Ab3) or anti- $\alpha$ tubulin antibodies (Ab-1; Oncogene) for IP and an anti-cJun antibody (sc-1694) for immunoblotting.

\section{In vitro GST pulldown assay}

The PCR fragments encoding the bZIP region of the AP1 proteins were fused in-frame with the GST portion in plasmid pGEX-2T (Pharmacia). The GST-bZIP proteins were expressed and purified according to the manufacturer's instruction, with the induction of the protein expression performed at $37^{\circ} \mathrm{C}$ for 3 $\mathrm{h}$. The fusion gene encoding FLAG-tagged BRCA1-AD in the pcDNA3 vector was also under the control of the bacteriophage $\mathrm{T} 7$ promoter. This plasmid was used for in vitro transcription and translation in the TnT Reticulocyte Lysate system (Promega). The ${ }^{35}$ S-labeled FLAG-BRCA1-AD was mixed with 10 $\mu \mathrm{g}$ of GST derivatives bound to agarose beads in $0.5 \mathrm{~mL}$ of binding buffer (50 mM HEPES at $\mathrm{pH} 8.0,150 \mathrm{mM} \mathrm{NaCl}, 1 \mathrm{mM}$ EDTA, $0.1 \%$ NP-40 and protease inhibitor tablets). The binding reaction was carried out at $4^{\circ} \mathrm{C}$ overnight and subsequently washed four times with the washing buffer (the same as binding buffer, except $0.5 \% \mathrm{NP}-40$ ), $30 \mathrm{~min}$ each round. The beads were eluted in $10 \mu \mathrm{L}$ of $2 \times$ protein sample buffer and the proteins were resolved on $10 \%$ SDS-polyacrylamide. The gel was then dried and exposed to X-ray films overnight.

\section{Real-time PCR}

The ABI PRISM 7700 Sequence Detection System (PE Biosystems) was used for the quantitative analysis of JunB mRNA expression. The following primers were designed using the Primer Express software provided by the manufacturer. JunB forward primer, ACTCATACACAGCTACGGGATACG; JunB reverse primer, GGCTCGGTTTCAGGAGTTTG; TagMan probe, 〈VIC〉CCCCTGGTGGCCTCTCTCTACACGATA〈MRA〉.

Primers, probes, and TagMan universal PCR master mixes were purchased from Applied Biosystems. PCR was performed in a $50-\mu \mathrm{L}$ reaction volume, using the default assay condition in the ABI PRISM 7700 Sequence Detector. The final concentra- tions for primers and probe were 300 and $250 \mathrm{nM}$, respectively. cDNA pairs (Clontech) normalized with $\beta$-actin and/or ribosomal protein S9 were used as the templates in the PCR reactions. In Figure 5D, pairs 1-4 come from HP101O, HP103O, $\mathrm{HP} 104 \mathrm{O}$, and HP105O; pairs 5-9 from a human ovary-matched cDNA pair panel (K1435-1); pairs 10-11 from HB102B and HP104B; and pairs 12-16 from a human breast-matched cDNA pair panel (K1432-1).

\section{Acknowledgments}

We thank Richard Baer and Ralph Scully for sharing reagents; Qinong Ye, Hongjun Zhong, and Anne Allison for technical assistance; Mark Alexandrow, Christian Loch, Sarah Aiyar, and Sagar Ghosh for critical reading of the manuscript; and members of the Li laboratory for stimulating discussion. This work was supported by grants from the National Cancer Institute and Department of Defense.

The publication costs of this article were defrayed in part by payment of page charges. This article must therefore be hereby marked "advertisement" in accordance with 18 USC section 1734 solely to indicate this fact.

\section{References}

Bakiri, L., Lallemand, D., Bossy-Wetzel, E., and Yaniv, M. 2000. Cell-cycle-dependent variations in c-Jun and JunB phosphorylation: A role in the control of cyclin D1 expression. EMBO J. 19: 2056-2068.

Bochar, D.A., Wang, L., Beniya, H., Kinev, A., Xue, Y., Lane, W.S., Wang, W., Kashanchi, F., and Shiekhattar, R. 2000. BRCA1 is associated with a human SWI/SNF-related complex: Linking chromatin remodeling to breast cancer. Cell 102: 257-265.

Chapman, M.S. and Verma, I.M. 1996. Transcriptional activation by BRCA1. Nature 382: 678-679.

Chinenov, Y. and Kerppola, T.K. 2001. Close encounters of many kinds: Fos-Jun interactions that mediate transcription regulatory specificity. Oncogene 20: 2438-2452.

Fan, S., Wang, J., Yuan, R., Ma, Y., Meng, Q., Erdos, M.R., Pestell, R.G., Yuan, F., Auborn, K.J., Goldberg, I.D., et al. 1999. BRCA1 inhibition of estrogen receptor signaling in transfected cells. Science 284: 1354-1356.

Fan, S., Ma, Y.X., Wang, C., Yuan, R.Q., Meng, Q., Wang, J., Erdos, A.M., Goldberg, I.D., Webb, P., Kushner, P.J., et al. 2001. Role of direct interaction in BRCA1 inhibition of estrogen receptor activity. Oncogene 20: 77-87.

Gayther, S.A., Warren, W., Mazoyer, S., Russell, P.A., Harrington, P.A., Chiano, M., Seal, S., Hamoudi, R., van Rensburg, E.J., Dunning, A.M., et al. 1995. Germline mutations of the BRCA1 gene in breast and ovarian cancer families provide evidence for a genotype-phenotype correlation. Nature Genet. 11: 428-433.

Harkin, D.P., Bean, J.M., Miklos, D., Song, Y.-H., Truong, V.B., Englert, C., Christians, F.C., Ellisen, L.W., Maheswaran, S., Oliner, J.D., et al. 1999. Induction of GADD45 and JNK/ SAPK-dependent apoptosis following inducible expression of BRCA1. Cell 97: 575-586.

Hu, Y.F., Hao, Z.L., and Li, R. 1999. Chromatin remodeling and activation of chromosomal DNA replication by an acidic transcriptional activation domain from BRCA1. Genes \& Dev. 13: 637-642.

Hu, Y.-F., Miyake, T., Ye, Q., and Li, R. 2000. Characterization of a novel trans-activation domain of BRCA1 that functions 
in concert with the BRCA1 C-terminal (BRCT) domain. I. Biol. Chem. 275: 40910-40915.

Johnson, R., Spiegelman, B., Hanahan, D., and Wisdom, R. 1996. Cellular transformation and malignancy induced by ras require c-Jun. Mol. Cell. Biol. 16: 4504-4511.

Karin, M., Liu, Z., and Zandi, E. 1997. AP-1 function and regulation. Curr. Opin. Cell Biol. 9: 240-246.

Lupas, A. 1996. Coiled coils: New structures and new functions. Trends Biosci. 21: 375-382.

Lupas, A., Van Dyke, M., and, Stock, J. 1991. Predicting coiled coils from protein sequences. Science 252: 1162-1164.

MacLachlan, T.K., Somasundaram, K., Sgagias, M., Shifman, Y., Muschel, R.J., Cowan, K.H., and El-Deiry, W.S. 2000. BRCA1 effects on the cell cycle and DNA damage response are linked to altered gene expression. I. Biol. Chem. 275: 2777-2785.

Mechta, F., Lallemand, D., Pfarr, C.M., and Yaniv, M. 1997. Transformation by ras modifies AP-1 composition and activity. Oncogene 14: 837-847.

Mechta-Grigoriou, F., Gerald, D., and Yaniv, M. 2001. The mammalian Jun proteins: Redundancy and specificity. Oncogene 20: 2378-2389.

Monteiro, A.N.A. 2000. BRCA1: Exploring the links to transcription. Trends Biosci. 25: 469-474.

Monteiro, A.N., August, A., and Hanafusa, H. 1996. Evidence for a transcriptional activation function of BRCA1 C-terminal region. Proc. Natl. Acad. Sci. 93: 13595-13599.

Neish, A.S., Anderson, S.F., Schlegel, B.P., Wei, W., and Parvin, J.D. 1998. Factors associated with the mammalian RNA polymerase II holoenzyme. Nucleic Acids Res. 26: 847-853.

Ouchi, T., Monteiro, A.N., August, A., Aaronson, S.A., and Hanafusa, H. 1998. BRCA1 regulates p53-dependent gene expression. Proc. Natl. Acad. Sci. 95: 2302-2306.

Pao, G.M., Janknecht, R., Ruffner, H., Hunter, T., and Verma, I.M. 2000. CBP/p300 interact with and function as transcriptional coactivators of BRCA1. Proc. Natl. Acad. Sci. 97: 1020-1025.

Parvin, J.P. 2001. BRCA1 at a branch point. Proc. Natl. Acad. Sci. 98: 5952-5954.

Passegue, E. and Wagner, E.F. 2000. JunB suppresses cell proliferation by transcriptional activation of p16(INK4a) expression. EMBO J. 19: 1969-1979.

Passegue, E., Jochum, W., Schorpp-Kistner, M., Mohle-Steinlein, U., and Wagner, E.F. 2001. Chronic myeloid leukemia with increased granulocyte progenitors in mice lacking JunB expression in the myeloid lineage. Cell 104: 21-32.

Rahman, N. and Stratton, M.R. 1998. The genetics of breast cancer susceptibility. Annu. Rev. Genet. 32: 95-121.

Scully, R. and Livingston, D.M. 2000. In search of the tumorsuppressor functions of BRCA1 and BRCA2. Nature 408: 429-432.

Scully, R., Anderson, S.F., Chao, D.M., Wei, W., Ye, L., Young, R.A., Livingston, D.M., and Parvin, J.D. 1997. BRCA1 is a component of the RNA polymerase II holoenzyme. Proc. Natl. Acad. Sci. 94: 5605-5610.

Shaulian, E. and Karin, M. 2001. AP-1 in cell proliferation and survival. Oncogene 20: 2390-2400.

Somasundaram, K., Zhang, H., Zeng, Y.X., Houvras, Y., Peng, Y., Wu, G.S., Licht, J.D., Weber, B.L., and El-Deiry, W.S. 1997. Arrest of the cell cycle by the tumour-suppressor BRCA1 requires the CDK-inhibitor p21WAF1/CiP1. Nature 389: $187-190$.

Welcsh, P.L. and King, M.-C. 2001. BRCA1 and BRCA2 and the genetics of breast and ovarian cancer. Hum. Mol. Genet. 10: 705-713.

Yarden, R.I. and Brody, L.C. 1999. BRCA1 interacts with com- ponents of the histone deacetylase complex. Proc. Natl. Acad. Sci. 96: 4983-4988.

Yu, X., Wu, L.C., Bowcock, A.M., Aronheim, A., and Baer, R. 1998. The C-terminal (BRCT) domains of BRCA1 interact in vivo with CtIP, a protein implicated in the CtBP pathway of transcriptional repression. J. Biol. Chem. 273: 25388-25392.

Zhang, H., Somasundaram, K., Peng, Y., Tian, H., Zhang, H., Bi, D., Weber, B.L., and El-Reiry, W.S. 1998a. BRCA1 physically associated with p53 and stimulates its transcriptional activity. Oncogene 16: 1713-1721.

Zhang, H., Tombline, G., and Weber, B.L. 1998b. BRCA1, BRCA2, and DNA damage response: Collision or collusion? Cell 92: 433-436.

Zheng, L., Li, S., Boyer, T.G., and Lee, W.H. 2000. Lessons learned from BRCA1 and BRCA2. Oncogene 19: 6159-6175.

Zheng, L., Annab, L.A., Afshari, C.A., Lee, W.-H., and Boyer, T.G. 2001. BRCA1 mediates ligand-independent transcriptional repression of the estrogen receptor. Proc. Natl. Acad. Sci. 98: 9587-9592. 


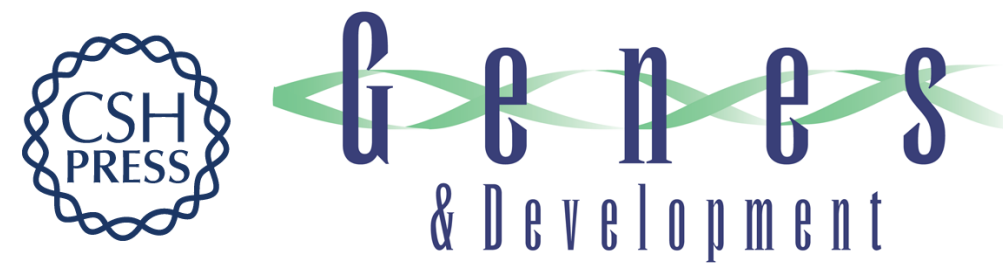

\section{JunB potentiates function of BRCA1 activation domain 1 (AD1) through a coiled-coil-mediated interaction}

Yan-Fen Hu and Rong Li

Genes Dev. 2002, 16:

Access the most recent version at doi:10.1101/gad.995502

References This article cites 36 articles, 15 of which can be accessed free at: http://genesdev.cshlp.org/content/16/12/1509.full.html\#ref-list-1

License

Email Alerting Receive free email alerts when new articles cite this article - sign up in the box at the top Service right corner of the article or click here.

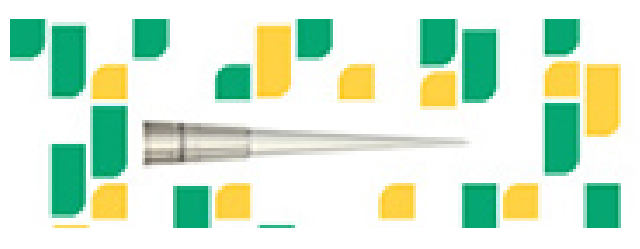

Focused on your science. 